medical illness. Prolonged EEG monitoring may be warranted after brief convulsive seizures to exclude NCSE. (Tay SKH, Hirsch LJ, Leary L et al. Epilepsia Sept 2006;47:1504-1509).

\title{
PANAYIOTOPOULOS SYNDROME: BENIGN AUTONOMIC EPILEPSY
}

The clinical features, EEG, misdiagnoses, and management of children with Panayiotopoulos syndrome (PS) are reviewed from Sophia Children's Hospital, GoudiAthens, Greece. Emesis is the predominant manifestation of autonomic seizures in PS. Other autonomic symptoms include pallor, mydriasis, urinary or fecal incontinence, and hypersalivation. One fifth have ictal syncope with or without convulsions. One half of the seizures last for $>30$ minutes, and constitute a nonconvulsive autonomic status. Two thirds of the seizures occur during sleep. The EEG shows multiple spikes, occipital predominating, and sometimes centro-temporal of Rolandic epilepsy. Prognosis is benign, one quarter of patients having a single seizure and half having 2 to 5 seizures. One fifth develop Rolandic or occipital seizures, remitting before age 16 years. Cardiorespiratory arrest during an autonomic seizure is a very rare occurrence. Misdiagnoses include acute encephalitis, syncope, migraine, benign Rolandic epilepsy, or gastroenteritis. Benzodiazepines are used to terminate nonconvulsive autonomic status, and prophylactic treatment is prescribed infrequently. Aggressive treatment is avoided because of risk of cardiorespiratory arrest. (Covanis A. Panayiotopoulos syndrome: a benign childhood autonomic epilepsy frequently imitating encephalitis, syncope, migraine, sleep disorder, or gastroenteritis. Pediatrics October 2006;118:1237-1243). (Respond: A Covanis MD, Neurology Department, Agia Sophia Children's Hospital, Thivon and Levadias, Goudi, 11527 Athens, Greece).

COMMENT. Vomiting as an ictal phenomenon has been described by various terms including ictus emeticus, diencephalic or autonomic epilepsy, abdominal epilepsy, and cyclic vomiting as a form of epilepsy (Millichap JG, Lombroso CT, Lennox WG. Pediatrics 1955;15:705-714; Freeman R, Schachter SC. Seminars in Neurology 1995;15:158-166; Ped Neur Briefs June and August 1995). Occipito-temporal spikes in the EEG, similar to those mentioned with PS, are described in previous reports of ictal vomiting. Are the symptoms described as PS sufficiently specific and novel to warrant classification as a separate syndrome?

\section{ANTIEPILEPTIC DRUGS}

\section{OXCARBAZEPINE MONOTHERAPY}

The efficacy of oxcarbazepine monotherapy in 60 children and adolescents (aged 6 months to 17.8 years; mean age 8.2 yrs) with partial onset epilepsy was evaluated by retrospective chart review in a study at St Christopher's Hospital for Children, Philadelphia, PA. Dosage ranged from 6 to $71 \mathrm{mg} / \mathrm{kg} /$ day (mean $26.3 \mathrm{mg} / \mathrm{kg} /$ day). The mean dose varied with age, higher doses were required in younger patients; in patients $<4$ years, the mean dose was $33.1 \mathrm{mg} / \mathrm{kg} /$ day; $8-12$ years, $25 \mathrm{mg} / \mathrm{kg} /$ day. Duration of therapy was 3 months to 8 years (mean duration 16.7 months). Reduction in seizure frequency was $>50 \%$ in $51(85 \%)$ 
patients, and complete control was achieved in $25(42 \%)$. Adverse events occurred in 10 $(16.7 \%)$ and included drowsiness, aggressive behavior, ataxia, dizziness, diplopia, and leg cramps. None had hyponatremia or skin rash. Of 24 patients switched from carbamazepine to oxcarbazepine because of toxic effects or poor seizure control, $79 \%$ had $>50 \%$ seizure reduction, and $37.5 \%$ became seizure-free. Serum levels were not determined. (Kothare SV, Khurana DS, Mostofi $\mathrm{N}$ et al. Oxcarbazepine monotherapy in children and adolescents: a single-center clinical experience. Pediatr Neurol Oct 2006;35:235-239). (Respond: Dr Kothare, St Christopher's Hospital for Children, Division of Pediatric Neurology; Director, Pediatric Epilepsy Program, Erie Avenue at Front Street, $3^{\text {rd }}$ floor ACP, Suite 3314, Philadelphia, PA 19134).

COMMENT. Oxcarbazepine monotherapy, a keto analog of carbamazepine, is effective and without serious side effect in children and adolescents with partial epilepsy. It is approved as monotherapy in children $>4$ years old and as adjunctive therapy in children $>2$ years old (in Europe, for $>6$ year-olds). The inverse relation between dose and age is a reflection of a more rapid clearance of oxcarbazepine in younger patients.

\section{VALPROATE-RELATED HYPERINSULINEMIA}

Changes in insulin metabolism during treatment with valproate (VPA) were investigated in a study of 51 patients receiving monotherapy ( 31 male and 20 female) and 45 healthy controls at University of Oulu, and Hospital of Children and Adolescents, University of Helsinki, Finland. VPA-treated patients had fasting hyperinsulinemia, while the fasting serum proinsulin and C-peptide levels did not differ from controls. Proinsulin/insulin and Cpeptide/insulin ratios were reduced in VPA-treated patients, and fasting plasma glucose levels were lower compared to controls. These changes were independent of the sex, seizure type, weight gain, and pretreatment weight, and were not due to weight gain or obesity. VPA does not induce insulin secretion but interferes with insulin metabolism in the liver, resulting in hyperinsulinemia and reduced plasma glucose. The obesity induced by VPA is secondary to the increase in circulating insulin. (Pylvanen V, Pakarinen A, Knip M, Isojarvi J. Characterization of insulin secretion in valproate-treated patients with epilepsy. Epilepsia Sept 2006;47:1460-1464). (Reprints: Dr V Pylvanen, Department of Neurology, PO Box 5000, 90014 Oulu, Finland).

COMMENT. VPA causes hyperinsulinemia and consequent fall in plasma glucose by interference with insulin metabolism in the liver. The hyperinsulinemia may then induce increased weight gain.

Serum total amylase, pancreatic amylase and lipase in VPA-treated epilepsy children. In a study of 23 patients, serum pancreatic amylase was decreased at 6 and 12 months treatment with VPA, whereas serum total amylase and lipase were unchanged. Nonpancreatic isoenzyme activities of amylase were higher at 6 or 12 months. Nonpancreatic amylase, probably derived from salivary glands, may be increased in children treated with VPA, and is not a reason to interrupt therapy. Measurement of serum pancreatic amylase and/or lipase is indicated when total amylase is increased without clinical symptoms of pancreatitis. (Voudris K et al. Brain Dev Oct 2006;28:572-575). 